\title{
EUROPEAN GUIDELINES FOR WORKPLACE DRUG TESTING IN ORAL FLUID
}

\author{
Michaela Brcak, a Olof Beck, b Tessa Bosch, ${ }^{\mathrm{b}}$ Duncan Carmichael, d \\ Nadia Fucci, e * Claire George, ${ }^{\mathrm{f}}$ Mark Piper, ${ }^{\mathrm{g}}$ Alberto Salomone, ${ }^{\mathrm{h}}$ \\ Wim Schielen, ${ }^{\text {i }}$ Stefan Steinmeyer, ${ }^{\text {, Sanna Taskinen, }}{ }^{\mathrm{k}}$ Weinmann, Wolfgang ${ }^{1}$
}

*Correspondence to : Nadia Fucci Institute of Public Health- Section of Legal Medicine - Catholic

University L.go F. Vito 1, 00168 Rome Italy.email: nadia.fucci@unicatt.it

a Greiner Bio-One GmbH, Kremsmünster, Austria

${ }^{b}$ Karolinska Institutet, Department of Laboratory Medicine, Stockholm, Sweden

c Department Clinical Pharmacology \& Toxicology, Hospital Pharmacy, MaastadLab, Rotterdam, The Netherlands

d AgriYork 400 Limited, Pocklington, York, United Kingdom

${ }^{e}$ Institute of Public Health-Section of Legal Medicine - Catholic University Rome Italy

$f$ Alere Toxicology Plc, Abingdon, Oxfordshire, United Kingdom

$g$ Randox Testing Services, Crumlin, Co. Antrim, United Kingdom

$h$ Centro Regionale Antidoping e di Tossicologia “A. Bertinaria”, Torino, Italy

i ELDC BV/ BeterVee BV, Kerkrade, The Netherlands

j Dräger Safety AG \& Co. KGaA, Lübeck, Germany

${ }^{k}$ VITA Laboratory Healthcare Services Ltd

${ }^{l}$ Institute of Forensic Medicine, University of Bern, Bern, Switzerland

\begin{abstract}
These guidelines for Legally Defensible Workplace Drug Testing have been prepared and updated by the European Workplace Drug Testing Society (EWDTS).

The European Guidelines are designed to establish best practice procedures whilst allowing individual countries to operate within the requirements of national customs and legislation. The EWDTS recommends that all European laboratories that undertake legally defensible workplace drug testing should use these guidelines as a template for accreditation. These guidelines are relevant to laboratory-based testing only. These guidelines follow current best practices and are constantly under review.
\end{abstract}

KEYWORDS: guidelines, oral fluid, EWDTS, workplace drug testing. 


\section{General \\ Introduction}

These guidelines represent an overview of the best practice for European laboratories providing oral fluid workplace drug testing services to maintain the legal defensibility of a drug test when the results, and the medical interpretation of the results are used in an employment disciplinary process, employment tribunal or a court of law. These guidelines are designed to ensure that the entire drug testing process is conducted to give accurate and reliable information about a donor's drug use.

The updated oral fluid guidelines have been communicated within the members of EWDTS, experts from different countries - and accepted by the board.

In the age of globalization the legal basis for workplace drug and alcohol testing has a complex structure consisting of international, European and national laws, acts and regulations from different levels (1). The European Workplace Drug Testing Society (EWDTS) has since 1988 offered a panEuropean platform to allow all sectors involved in Workplace Drug Testing (WDT) to share best practices.

Since 2010, first guidelines published with the aim to provide a "handbook" for specimen collection, analysis of the specimen and interpretation of the results. Since that time there have been goals and specific directives which have impacted the WDT sector. This will continue with the impact of further data protection and human rights legislation, for example, GDPR ( General Data Protection Regulations). The GDPR (EU- 2016/679) is a regulation by which the European Parliament, the Council of the European Union and the European Commission intend to strengthen and unify data protection for all individuals within the European Union (EU). In many European countries, there is no legal mandate to test, and conversely there is no legislation to prevent a test programme.

Most countries have implemented a testing regime in safety critical workplace sectors, e.g. nuclear, transportation, refinery operations, and of course testing programmes within the military and prison systems are prevalent in many countries.

Most of the European countries have no specific legislation on WDT. In Belgium, Greece, Slovenia, Sweden and Luxembourg further Acts like "Law on Safety and Health at the Workplace, Public Employment Act, Code for civil servants" and others permit pre-employment testing or occupational physicians are authorized to make examinations when required for specific jobs.

In the Czech Republic, Estonia, Ireland, Lithuania, Slovakia WDT is regulated in the Labour Law or Safety, Health and Welfare at Work Acts or similar acts. In Italy since 2007 WDT is established and prescribed in the "Decree on Health and Safety at Work (81/08)" for jobs which bring out safety hazards to others (5). The Act on Workplace Drug Testing permits WDT in Finland. By contrast there is no legislation and only alcohol testing can be done in Hungary: "The Act on Labour Safety (No. 93/1993) does not authorize the labour safety controllers to make drug tests." In Austria, the "Employee Protection Act" defines that the "employee is obliged not to be in a state caused by the use of alcohol, medicines and drugs that endanger themselves or others" - on one's own authority. In Portugal, Germany the responsibility for workers' safety and health is part of the employers' duties.

This "duty of care" requirement is common in many countries, and in turn. This mandate can be used to initiate a testing protocol.

Regarding the hierarchy of jurisprudence and all the different national laws it is necessary to set up additional recommendations and to bring in line WDT procedures which were already common in 
practice. Otherwise companies and institutions may not be able to implement the same procedure in all its offices, whether or not the headquarters are based in Europe. EWDTS attempts to ensure that WDT in Europe is performed to a defined quality standard and in a legally secured way. The three main points all EWDTS guidelines are collection of the specimen, analysing the specimen and interpretation of results. Some chapters handle with general aspects like "Laboratory Organisation", "Quality Assurance and Quality Control” and these parts are identical in Urine and Oral fluid guidelines.

In the Oral fluid guidelines collection devices, collection procedures, chain of custody and validity testing are reported. As there are different kits on the market - the focus is on oral fluid collection devices, the components of the whole collection kit and the process of oral fluid collection. There should be a guarantee that all oral fluid collection devices fulfil the requirement for exact determination of known volume. There is the demand for precise determining of the oral fluid amount in the collected sample to ensure accurate calculation of drug concentrations. The use of "two sample containers, demonstrably clean and unused" as well a "tamper-evident seal for each container" are further requests. In the subchapter "Oral Fluid Collection Procedures" the demand for A and B samples is described. The measurement of endogenous biomarker in accordance with reference values, the accurate known dilution factor and sample volume are strongly recommended to verify the collected sample. Any uses of adulterants and efforts to tamper samples by the donors have to be uncovered forcefully. In summary the updated version of the European Guidelines for Workplace Drug Testing in Oral fluid are geared to bring together different methods of operation and to ensure best practice procedures.

\section{Objectives}

- $\quad$ To provide a common framework for European providers of oral fluid workplace drug testing services.

- To promote standards by providing guidelines which are accepted at a European level.

- To ensure that the processes undertaken are capable of legal scrutiny.

- To provide safeguards to protect the dignity of the specimen donors and the validity of the specimen.

- To define for laboratories common quality assurance and quality control criteria that are capable of being accredited by an external body.

- To ensure that the entire drug testing process is conducted to give accurate and reliable information about drug use of the donor.

\section{Scope}

These guidelines consider the three key stages of the workplace drug testing process.

- Specimen collection: obtaining the oral fluid specimen from the donor

- Laboratory analysis: analysis of the sample for the presence of drugs

- Interpretation: review and interpretation of the analytical results

\section{Service Provision}

Where a service provider is contracted to deliver all the stages, they must ensure that the minimum criteria in this document are met in all the key areas.

In those instances where a customer may undertake some stages of the process within their own organisation (e.g. specimen collection or interpretation), the service provider has a 'duty of care' to ensure that the customer understands the full implications of the drug testing process. 
The service provider does not have the authority to make decisions regarding the fitness for work of any individual being tested. It is recommended that any issues related to fitness for work be referred to the company's medical representative.

\section{Drug testing in Context}

It should be explained to any purchaser of a laboratory drug testing service that drug testing should form part of an overall drug policy, which the purchaser has agreed with his employees and should have in place before testing is initiated.

The service provider should have an effective company drugs policy in place. The policy may include drug testing of the staff involved in the analysis and reporting of workplace drug testing results.

\section{Outline of drug testing process}

\section{Specimen collection}

Oral fluid specimens for legally defensible drug testing need to be collected under circumstances which respect the dignity of the individual whilst ensuring that the specimen is freshly collected Suitable records must be made when the specimen is collected to document that the specimen collected and the specimen received by the laboratory is one and the same.

This is the first link in the chain of custody process which, when reconstructed at a later date, can be used to document that the final result belongs to the specimen collected.

\section{Analysis}

When the specimen is received at the laboratory, checks on the integrity of the specimen are carried out. Providing the specimen passes the integrity checks a portion of the specimen is taken and screened for the presence of drugs and sample validity. If the screen results are all negative no further analysis is necessary.

However if the screen tests carried out indicate the possible presence of a drug (above a predefined cut-off level) a confirmation test to prove or disprove the presence of the drug or drug metabolite indicated by the screening test must be carried out on another portion of the specimen.

When a negative result is obtained, either after the screen or confirmation test, it can be reported to the customer. Positive results may require interpretation.

\section{Interpretation}

A laboratory positive result may be due to other reasons than intake of illicit drugs (i.e. prescribed, over-the-counter medication or dietary causes). It requires interpretation that is best carried out by the laboratory toxicologist in conjunction with a qualified medical practitioner who can consult both with the donor and the donor's medical practitioner.

\section{Record keeping}

Suitable records must be made during the analytical process to document that the specimen received by the laboratory and the specimen, about which the final report is written, are one and the same. All samples which prove positive for the presence of drugs, and all records of the analytical process, must be kept for an agreed period of time or according to national legislation to allow for any challenges to be made regarding the findings. 
If the customer requires an independent toxicological review, the laboratory must make available, if requested, the analytical data upon which it based its final report. In table 1 the definitions adopted for these guidelines are reported.

\section{Oral Fluid Collection}

\section{Introduction}

This is the first link in the chain of custody process which, when reconstructed at a later date, can be used to prove that the final result belongs to the specimen collected.

The collection process must be carried out by someone formally assessed as competent and authorised to carry out the collection. Standard Operating Procedures (SOP) must be written for the collection process, the storage of collection devices, the training of Collecting Officers and the shipping of the collected specimen to the laboratory. These procedures must be followed precisely. Collection procedures must cover the following aspects:

- $\quad$ Privacy and security of the specimen collection site

- $\quad$ Steps to ensure that the specimen collection is supervised

- Steps to protect against tampering and adulteration

- Identification of the donor giving the specimen

- Evidence of the written informed consent of the individual to the analysis of the specimen (an example is given in Appendix B)

- Disclosure of recent medication, or evidence that the individual was advised of the significance of recent medication

- All information is considered as confidential.

All specimens for legally defensible drug testing must be collected under circumstances that respect the dignity of the individual whilst ensuring that the specimen is freshly generated and has not been tampered with in any way. The collection site must be secure and the absence of potential interfering substances must be guaranteed. The validity of the specimen has to be guaranteed.

Suitable records must be made when the specimen is collected to prove that the specimen collected and the specimen received by the laboratory is one and the same.

Where the customer takes responsibility for the collection process, the service provider has a duty of care to ensure that these guidelines are understood.

\section{Personnel}

Specimens must be collected by suitably trained personnel (Collecting Officers). Although no healthcare professional education is required, documented training, which includes a demonstration of competence, must be undertaken before collections are performed.

The training must include, at a minimum, instructions on the following:

- The collection process

- The storage and transport conditions of samples

- The chain-of-custody process

- Troubleshooting (i.e. how to deal with issues like refusal of the test, insufficient sample, suspicion of tampering of the sample, dry mouth)

- The responsibility of the collecting officer for maintaining donor privacy, confidentiality of information, and specimen integrity. 
- Ethical issues, especially regarding the declaration by the donor of the present use of prescribed medications.

On successful completion of collector training a person may begin performing collections.

\section{Oral Fluid Collection Kits}

The laboratory and manufacturer must demonstrate that the device in no way impairs the ability of the laboratory to detect the drugs at the cut-off levels recommended in these guidelines. It is recommended that the device used to collect the oral fluid sample collects a known volume. This may be achieved through spectrophotometrically determination or through an integrated volume indicator. In case of uncertainty gravimetric analysis is strongly recommended. Scientific data which demonstrate the accuracy of the volume of any buffer (if used) and collected sample have to be available. The laboratory should be able to clearly identify which collection device has been used to collect the sample.

The collection kit should comprise the following components:

- $\quad$ Specimen collection device(s).

- Chain of custody donor form.

- A unique identifier that links the chain of custody form and sample containers.

- At least two sample containers, demonstrably clean and unused.

- Tamper-evident seal for each container.

- Packaging components that satisfy current postal and courier regulations.

\section{Chain of Custody Forms}

The minimum information required on the Chain of Custody Form is:

- Unique identification to link the form to the specimen container(s) (typically a barcode label or code number assigned to the sample).

- Information uniquely identifying the donor.

- Evidence that the donor identity has been confirmed.

- Evidence that the donor has given informed consent for the specimen to be tested.

- Date, time and place of collection.

- Names and signatures of all individuals who had custody of the specimen during the collection process.

- The opportunity to record any medication prescribed or non-prescribed that may have been taken in the days prior to the specimen being collected.

- Copies for donor, employer and laboratory.

\section{Oral Fluid Collection Procedures}

One sample is collected and then split in the presence of the donor into two separate containers labelled sample A and sample B.

It is acknowledged that currently some oral fluid collection devices cannot mechanically collect and generate two separate samples from the single collection procedure. In this case, two devices may be used to generate two samples. As these samples are discrete and not homogeneous the aliquots could be mixed and then divide into two aliquots (A and B).The sequential collection should be done within 5 minutes away from each other. In addition the exact times of the generation of the 
samples must be noted in the donor consent documentation. The two samples are then sent to the testing facility. Regardless of which type of device is used, there must be a "volume adequacy indicator" on the device to show to the collector that an adequate minimum volume has been collected, if two separate collection procedures are used to generate two samples. The receiving laboratory must gravimetrically or spectrophotometry determine a more precise quantity of collected oral fluid present in the container. When gravimetrical determination is used to measure collected oral fluid it is recommended to determine average net-weight of unused collection devices for each batch. This known amount of oral fluid is very important in the subsequent calculation of drug concentrations and individual manufacturer's tolerances must be known and confirmed. In all cases the testing laboratory should receive two samples, labelled A and B. An example oral fluid collection protocol is detailed in Appendix A.

\section{Laboratory Organization}

A Quality Management System of the organization / laboratory is required via accreditation according to EN ISO/IEC 17025 and/or EN ISO 15189 in fields of forensic toxicology and/or workplace drug testing analysis.

All personnel must have contracts with the institution (Laboratory Organisation) which they work for and every person must have agreed to the "confidentiality policy" of the institution (in written form).

\section{Personnel}

All personnel should adhere to the requirements of EN ISO/IEC 17025 and/or EN ISO 15189 International Standards and as such, only staff who are suitably qualified and whose competence has been formally assessed can work within the laboratory. The laboratory must maintain accurate job descriptions for managerial, technical and key support personnel involved in the analytical tests. The laboratory must keep records that establish the individual's qualifications / competency for all functions performed. The individual's file must include an up-to-date curriculum vitae listing educational qualifications and previous employment experience, training and competency assessment records for the current tasks performed. Personnel performing specific tasks shall be qualified on the basis of appropriate education, training, experience and/or demonstrated skills, as required. All laboratory personnel must have received training in Health and Safety issues, the Control of Substances Hazardous to Health (COSHH) Regulations and other relevant legislation.

The key functions outlined below are identified as the minimum requirement for a laboratory to maintain EN ISO 17025 and/or EN ISO 15189 accreditation for the provision of workplace drug testing services and/or forensic toxicology. It is acceptable for individuals to have responsibility to carry out more than one role. By virtue of the laboratory's accreditation, it can be accepted that the appropriate qualifications for each role are in place. Role titles may vary between organisations, but the responsibilities will remain the same.

\section{Laboratory Security}

Drug testing laboratories must have a robust security system to ensure that no unauthorised personnel gain access to the laboratory processes or to areas where samples or records are stored as mentioned in EN ISO 17025 and/or EN ISO 15189.Unescorted access to these secured areas must 
be limited to authorised individuals. The laboratory must maintain a record that documents the entry and exit of all visitors to the secured laboratory areas. The laboratory must maintain a record of all staff who are authorised to enter the secure laboratory areas. This list must be reviewed and updated on a regular basis. Sample containers must be retained within the secure laboratory area until the disposal date.

\section{Laboratory Director}

There must be one person who has overall responsibility for the professional, organisational, educational, and administrative activities of the drug testing facility.

This person is responsible for the day-to-day management of the drug testing laboratory.

Some of the functions may be delegated to other appropriately qualified personnel but the overall responsibility for any delegated functions will remain with the designated Laboratory Director (typically the Laboratory Supervisor).

\section{Authorizing Scientist}

A person responsible for the review and certification of pertinent data and quality control results, prior to release of accurate and reliable analytical results.

\section{Laboratory Analyst}

A person responsible for undertaking the day-to-day analytical procedures.

\section{Toxicologist}

A person responsible for interpreting a toxicological analytical result for the customer or the customer's designated Medical Review Officer.

\section{Expert Witness}

A person to present evidence to administrative or disciplinary proceedings that are based on analytical results reported by the laboratory.

\section{Quality Manager}

A person responsible for quality assurance within the laboratory organisation.

\section{Other Personnel}

Other technical or non-technical staff who must have the necessary training and skills for the tasks assigned.

\section{Laboratory Analysis Procedures \\ Process}

When specimens are received at the laboratory, initial checks on the sample's chain of custody and appearance are carried out. If the specimen passes these checks a portion of the specimen in container " $A$ " is taken and goes through initial screening tests for the presence of drugs. Further testing of sample validity may also take place at this point. If the screening results are all negative (below a pre-defined cut-off level) no further analyses are necessary. However if the screening tests carried out indicate the possible presence of a drug (above a pre-defined cut-off level) a 
confirmation test to prove or disprove the presence of the drug or drug metabolite indicated by the screening test must be carried out on another portion of the specimen.

The screen-only presumptive positive test is not considered to be legally defensible, but may report preliminary presumptive positive results to the clients as local legislation allows. In the report it has to be mentioned that preliminary presumptive positive results need confirmation. If the first analysis is performed by a confirmation-level analysis (mass spectrometry), the positive findings have to be retested with another portion of the sample.

\section{Chain of Custody}

Laboratories must use chain of custody procedures to maintain control and accountability of specimens and aliquots from receipt through completion of testing, reporting of results, during storage, and continuing until final disposal of specimens and aliquots.

Chain of custody records must be maintained on paper or in computerised form.

\section{Sample Receipt}

The laboratory should receive at least two sealed sample containers and a corresponding chain of custody form. At least one of these (referred to, in this document, as the " $\mathrm{B}$ " container) must be retained unopened and stored in conditions that reflect the storage of the sample under test (referred to, in this document, as the "A" container).

When a sample is received in the laboratory:

- Incoming orders and samples must be registered by the laboratory.

- Incoming samples are immediately checked regarding completeness, intactness and suitability for testing.

- Its packaging must be examined for evidence of tampering in transit.

- The information on the sample containers within the package must be compared with the information on the accompanying chain of custody form.

- Any discrepancies must be noted and, where appropriate, reported immediately to the customer. Some minor discrepancies may be tolerated in the documentation without termination of the analysis. These must be agreed with the customer prior to analysis and should be documented.

- Appendix C lists examples of fatal flaws in the chain of custody and is provided for guidance. Flaws of this nature would normally result in the sample not being tested.

\section{Sample Processing}

Separate representative portions (aliquots) of the sample in container 'A' will be used for the screening and confirmation tests. The sample preparation should follow the standard operating procedure and the manufacturer's instructions for the collection system being used. Aliquots must be taken in such a manner that excludes the possibility of contamination.

Short-term storage: samples that are not currently undergoing analysis must be refrigerated at 2$8^{\circ} \mathrm{C}$. Stability has to be investigated and appropriate measures undertaken to ensure the sample is valid for the analysis. The long term storage conditions are device dependent and the conditions should be as recommended by the device manufacturer.

The A and the B samples must be stored under identical conditions.

The quality control requirements must be satisfied when conducting either screening or 
confirmation tests, either on single samples or samples grouped in batches.

\section{Oral Fluid Validity Testing}

The aim of validity testing is to demonstrate that the sample submitted for analysis is oral fluid. The validity of the sample must be checked either before or during the screening process. The minimum validity test that must be completed for oral fluid is the visual inspection of the sample(s), measurement of oral fluid volume and testing on matrix authenticity through measurement of endogenous biomarkers (e.g. salivary amylase, cortisol ...). The laboratory may also test for adulterants and sample tampering.

Following data have to be coherent:

- Collection time

- Dilution factor (gravimetrical analysis or measured spectro-photometry)

- Sample volume

- Endogenous biomarker in accordance with reference values (e.g. salivary Amylase, Cortisol...)

\section{Testing for Adulterants}

Additional validity tests should be considered when the following conditions are observed:

- Abnormal physical characteristics (e.g., unusual colour or texture for the specific used device through bleeding, unusual odour, missing “pads”, ... )

- Reactions or responses characteristic of an adulterant obtained during initial or confirmatory drug tests (e.g., non-recovery of internal standards, unusual response); or possible unidentified interfering substance or adulterant.

A sample should be reported as e.g. "Sample cannot be used for screening and/or confirmation analysis" in the following situations:

- A valid immunoassay drug test result cannot be obtained on two separate aliquots (e.g. a reasonable suspicion on interference occurred).

- A valid drug test result cannot be obtained on two separate aliquots with drug confirmatory assay (a reasonable suspicion on interference occurred - e.g. components in the buffer which might interfere or cause ion suppression).

- The physical appearance of the sample is such that testing the sample may damage the laboratory's instruments.

- Gravimetrical analysis exposes unexplainable deviation of weight (i.e. less weight caused by "sucked pads"; higher weight through water in the mouth.)

- Spectrophotometrical analysis and measurement of endogenous biomarker indicates a dilution of the sample with water. 


\section{Analytical Methods and Validation \\ Acceptable Screening Techniques}

Following methods are accepted e.g.:

- Immunoassays

- Gas Chromatography

- $\quad$ Liquid Chromatography as UPLC/UFLC

- All chromatographic techniques coupled to mass spectrometry.

- Capillary Zone Electrophoresis

\section{Laboratory Screening Tests}

The initial screening test must use an appropriate technique. The assay using the selected technique must be validated prior to its use.

Recommended maximum screening calibration cut-off concentrations for workplace drug testing are listed in TABLE 2. These recommended cut-off concentrations may be subject to change reflecting advances in technology and knowledge.

Cut-off concentrations for substances not indicated in TABLE 2 will need to be agreed with the customer taking into account the performance of the assays to be used and the pharmacokinetics of the drugs involved.

All screening test results must be reviewed with regard to the results of the validity tests performed. Samples that test negative on all the initial screening tests and pass the validity tests must be reported as negative and the samples can be disposed of as agreed with the customer.

Samples that test negative on all the initial screening tests but fail the validity tests may be further investigated to determine the reason why.

The presumptive presence for any drug following the initial preliminary screen must have the presence of the drug or drug metabolite confirmed (refer to section Confirmation Tests). If the first analysis is performed by a confirmation-level analysis (mass-spectrometry), the positive findings have to be confirmed and quantified by reanalysis with another portion of the sample.

\section{Standardization of Laboratory Screening Assays}

All assays must be calibrated against appropriate standards by following laboratory protocols based on the manufacturer's recommendations or validated alternatives. The assay must be calibrated against one named compound, and the cross-reactivity to other related compounds must be determined. The customer must be informed of the limitations of the tests.

\section{Confirmation Tests}

The presence of the drugs indicated by a positive screening result must be confirmed using a chromatographic technique in combination with mass spectrometry (e.g. GC-MS or LC-MS). If the first analysis is performed by a confirmation-level analysis (mass spectrometry), the positive findings have to be confirmed and quantified by reanalysis with another portion of the sample.

All confirmations must be quantitative. The customer must be informed of the compounds detected in the confirmation tests. Recommended maximum confirmation cut-off concentrations for workplace drug testing are given in TABLE 3. The cut-off concentrations are a result of modern instrumentation techniques and the relatively short detection time window in oral fluid matrices. These cut-offs are the maximum recommended cut-offs for workplace drug testing purposes. 
Confirmation cut-off concentrations may be subject to change as advances in technology or other considerations warrant identification of substances at other concentrations.

Confirmation cut-off concentration for substances not indicated in Table3 must be agreed with the customer taking into account the performance of the assays to be used and the pharmacokinetics of the drugs involved. Samples that are below the agreed cut-off concentration must be reported negative. No further testing will be undertaken and the samples may be discarded as per the customer agreed timetable.

Samples that contain drugs and/or metabolites at concentrations greater than or equal to the agreed cut-off level must be reported positive.

Laboratories must adhere to national and international guidelines that specify additional criteria for chromatographic and mass spectral acceptability.

\section{Validation}

All methods must be validated and their suitability for intended purpose must be evaluated in accordance with EN ISO/IEC 17025. Laboratories accredited according to EN ISO 15189 should produce evidence of adequate method validation and certificate of participation to Proficiency Test and External Quality Assessment.

The following parameters have to be determined at least for quantitative confirmation analyses and whenever possible, for screening analyses: precision, cut-off verification, selectivity, limit of detection, limit of quantification, sensitivity, specificity, stability, measurement uncertainty, recovery of the collection device and matrix effects.

\section{Authorization and Reporting of Results}

Before any laboratory test result is released, the results must be reviewed and certified as accurate by a competent member of staff (analytical validation).

At a minimum, the report must include the specimen identification number and the test result (positive/negative) for each sample submitted. Reporting must be managed in accordance with EN ISO/IEC 17025 and/or EN ISO 15189 requirements. In addition the cut-off used for the test should be included. Only drugs that have been confirmed by a recognised confirmation test can be reported as positive. Samples that fail integrity or validity tests must be identified to the customer on the report. The laboratory must define and agree the meaning of all terms used in the report to the customer. Results must be transmitted to the customer's designated representative in a manner that will ensure confidentiality of the information. Laboratory results should not be provided verbally. Written or electronic results must be transmitted to the customer's designated representative in a manner that will ensure confidentiality of the information.

\section{Long-Term Storage of Samples}

The laboratory must demonstrate that the long term storage conditions of samples are adequate to ensure that analytes are stable over the time period required for any re-test. Currently long-term deep frozen storage $\left(-20^{\circ} \mathrm{C}\right.$ or below) indicates that most positive samples will remain suitable for any necessary retest. Unless otherwise authorised in writing by the customer, drug testing laboratories must retain all samples confirmed positive in properly secured long-term frozen storage for a minimum of 1 year. Within this one-year period the customer may request the laboratory to retain the sample for an additional period of time. If no such request is received, the laboratory may 
discard the sample after the end of 1 year. The laboratory shall be required to maintain any samples known to be under legal challenge for a further agreed period. Samples must be retained within the secure laboratory area until the disposal date agreed with the customer. Negative samples $(A+B)$ samples may be discarded as per the laboratory and customer agreed timetable.

\section{Records}

The laboratory must maintain and make available for an agreed period, documentation of all aspects of the testing process involved in the generation of a positive result.

The required documentation must include:

- Training and competency records for all individuals authorised to have access to samples and sample data.

- Chain of custody forms

- Quality assessment/quality control records

- Standard operating procedures

- All test data (including method validation, calibration curves and calculations for determining test results)

- Maintenance and instrument calibration records

- Reports

- Records of proficiency testing and computer generated data

The laboratory will be required to maintain documents for any sample under legal challenge for a further agreed period.

Document control must be managed in accordance with EN ISO/IEC 17025 and/or EN ISO 15189 requirements and records containing details of individuals should be dealt with in line with European Data Protection Legislation.

\section{Quality Assurance and Quality Control}

Quality Assurance

Drug testing laboratories must have a quality management system which encompasses all aspects of the testing process including but not limited to:

- $\quad$ Sample receipt

- Chain of custody

- $\quad$ Security and reporting of results

- Screening and confirmation testing

- Certification of calibrators and controls

- Validation of analytical procedures

Quality assurance procedures shall be designed, implemented and reviewed to monitor the conduct of each step of the testing process.

The testing laboratory and all screening and confirmation tests used in workplace drug testing must be fully validated and accredited by a recognised external accreditation body.

When an unaccredited method is used the customer should be informed accordingly. 


\section{Quality Control}

Calibrators and controls shall be prepared using either certified drug reference materials or certified standard solutions obtained from where possible two commercial manufacturers and should be appropriate to the matrix. If two manufacturers are not feasible then the controls should be taken from separate lots from the same manufacturer.

The laboratory must retain records to demonstrate that all calibrators and controls are traceable back to primary standards (if available). The calibrators and controls shall be properly labelled as to content, concentration, data placed in service and expiry date. All standards (e.g. pure reference materials, stock standard solutions, purchased standards) shall be labelled with the following:

- $\quad$ Date received (if applicable)

- Date prepared or opened or placed in service

- Expiration date

- Initials of the technicians who has prepared the (in house) calibrator etc.

All data acquired on control samples as well lot number of drug reference materials must be recorded in such a way as to facilitate interpretation of control results and trends.

\section{Laboratory Screening Tests}

These are the minimum requirements for the suitable control of all laboratory screening tests. A system suitability check must be carried out prior to the analysis of samples.

Screening tests are carried out using reagents and calibrators directly provided by the manufacturers provided the analysis is carried out according to the indications and the value of cut-off defined by the manufacturer. Since the negative outcome of a screening analysis is generally accepted as valid, it is essential to verify that the method can minimize false negative results.

Assays must be calibrated weekly or when quality control samples indicate poor performance.

Control samples at concentrations of approximately $50 \%$ below and above the cut-off concentration for each drug group must be included in every batch of samples. These must be sourced independently from calibrators. Quality control samples must comprise at least 5\% of the total number of samples in each batch being analysed.

\section{Confirmation tests}

These are the minimum requirements for identification of analytes and confirmation of results.

\section{Identification}

\section{Mass spectrometry coupled to chromatography}

a) Mass spectrometry coupled to a chromatographic separation method is a very powerful combination for identification of an analyte in the sample extract. It simultaneously provides retention time, ion/charge ratios and relative abundance (intensity) data.

\section{Requirements for chromatography}

b) The minimum acceptable retention time for the analytes under examination should be at least twice the retention time corresponding to the void volume of the column. The retention time of the analytes in the extract should correspond to that of the calibration standard (may need to be matrixmatched) with a tolerance of $\pm 1 \%$, for both gas chromatography and liquid chromatography. 
Tolerance $1 \%$ means (with rt: retention time):

$$
\frac{r t_{\text {analyte in sample }}-r t_{\text {analyte in calibrator }}}{r t_{\text {analyte in calibrator }}} \leq 0.01
$$

\section{Requirements for mass spectrometry (MS)}

c) Reference spectra for the analytes should be generated using the same instruments and techniques used for analysis of the samples. If major differences are evident between a published spectrum and the spectrum generated within the laboratory, the latter must be shown to be valid.

d) Identification relies on proper selection of diagnostic (characteristic) ions. The (quasi) molecular ion is a diagnostic ion that should be included in the measurement and identification procedure whenever possible. In general, and especially in single-stage MS, high $m / z$ ions are more specific than low $m / z$ ions (e.g. $m / z<100$ ).

e) Extracted ion chromatograms of sample extracts should have peaks (exceeding S/N 3:1 of similar retention time, peak shape and response ratio to those obtained from a calibration standard analysed at comparable concentration in the same batch. Shift in retention time should not exceed 1\% compared to calibration standard. Chromatographic peaks from different selective ions for the same analytes must overlap with each other. Where an ion chromatogram shows evidence of significant chromatographic interference, it must not be relied upon to quantify or identify residues. The ion that shows the best signal-to-noise ratio and no evidence of significant chromatographic interference should be used for quantification.

f) In case of full scan measurement, careful subtraction of background spectra, either manual or automatic, by deconvolution or other algorithms, may be required to ensure that the resultant spectrum of the chromatographic peak is representative. Whenever background correction is used, this must be applied uniformly throughout the batch and should be clearly indicated.

g) Different types and modes of mass spectrometric detectors provide different degrees of selectivity and specificity, which relates to the confidence in identification. General requirements for identification by MS-methods have been published and should be regarded as guidance criteria for identification, not as absolute criteria to prove presence or absence of a compound.

h) The relative intensities or ratios of selective ions (full-scan MS or SIM) or product ions (MS/MS), expressed as a ratio relative to the most intense (product) ion, should correspond to those of the calibration standard at comparable concentrations and measured under the same conditions. Matrix-matched calibration solutions may need to be used. Table 4 indicates the maximum tolerances for ion ratios (6).

i)The variability of ion ratios should preferably be determined from calibration standards during initial method validation and subsequently during routine analysis. Diagnostic ions should have an ion ratio of $>0.05$ (least/most intense ion). 
j) At higher deviation of the relative abundance of a qualifier ion, analysis needs to be repeated. If tolerances still remain beyond acceptance criteria, further investigation of influences of matrix effects or disturbing compound are recommended - such as standard addition experiments or chance of chromatographic system - to verify or to exclude the presence of a compound. In these cases complementary interpretation by an experienced analyst is recommended.

k) For a higher degree of confidence in identification, further evidence may be achieved from additional mass spectrometric information. For example, evaluation of full scan spectra, isotope pattern, adduct ions, additional accurate mass fragment ions, additional product ions (in MS/MS), or accurate mass product ions. For High resolution (HRMS) the mass resolution shall typically be greater than 10000 for the entire mass range at $10 \%$ valley (which equates to resolving power of 20000 FWHM (full width at half maximum). For accurate mass measurements (AMM) the instrument mass error in routine mass measurements must be less than $2 \mathrm{mDa}$ and resolution shall typically be greater than 5000 FWHM.

For combination of LC with HR-QToF-MS/MS or other mass spectrometric technique with HR-MS/MS the following settings are recommended for mass spectral identification based on a mass spectral library (commercial or home-made):

For HR-QToF-MS/MS analysis a mass resolution of $>10000 \mathrm{amu}$ with a mass accuracy of $<5 \mathrm{ppm}$ and an isotope ratio comparability of better than $80 \%$ should be used in routine analysis. If comparison to a library is performed with MS/MS spectra, a fit value of $>70 \%$ should be used as a threshold, for identification of compounds. Additionally, 1\% tolerance of LC-retention time is required (see above, e).

l) The chromatographic profile of the isomers of an analyte or any relevant metabolites may also provide evidence. Additional evidence may be sought using a different chromatographic separation system and/or a different MS-ionisation technique.

\section{Confirmation of results}

If the initial analysis does not provide unambiguous identification or does not meet the requirements for quantitative analysis, a confirmatory analysis of the A-sample is required. This may involve reanalysis of the extract or the sample. In cases where a detection threshold for a drug is exceeded, a confirmatory analysis of a portion of the B-sample may be required by the authorities. For unusual analyte/matrix combinations, a confirmatory analysis is also recommended.

The use of different determination techniques and/or confirmation of qualitative and/or quantitative results by an independent expert laboratory will provide further supporting evidence.

\section{External Quality Assessment}

The laboratory must take part in appropriate external quality assessment schemes. When the scheme is not available, the laboratory has to conduct appropriate inter-laboratory testing to ensure appropriate assay performance.

\section{Sub-contracting}

Drug testing laboratories should carry out all laboratory work with their own personnel and equipment. If it is necessary to sub-contract, inter-laboratory transfer of samples is performed with 
strict adherence to chain of custody procedures. The sub-contracted laboratory and its methods must be accredited by a recognised external accrediting body and compliant with these guidelines. Analyses undertaken by sub-contracted laboratories must be identified on the test report to the customer.

\section{Interpretation of Results}

A confirmed analytical positive result may be due to medication (prescribed or over-the-counter) or due to dietary causes. An essential part of the drug testing process is the final review of analytical results. The interpretation is best carried out by a qualified medical professional (e.g. Medical Review Officer) or a Forensic Toxicologist (depending on the country-specific situation).

\section{Toxicology Review}

It is mandatory that a toxicologist is available to advise the customer and/or Medical Review Officer regarding queries with test results.

\section{Medical Review}

The Medical Review Officer (MRO) is a medical physician with responsibility for interpreting laboratory results together with a toxicologist. Depending on the country-specific situation a medical physician usually has greater access to medical records than a toxicologist and may therefore be in a better position to provide interpretation of positive analytical results.

The MRO must have specialist knowledge of and training in

- specimen collection procedures,

- analytical procedures,

- chain of custody and

- alternative explanations for positive analytical results.

The MRO can issue a negative report for a positive analytical result if the test result is likely to be due to the use of declared medication, or a valid alternative medical explanation has been found.

The service provider may provide access to an independent medical review service.

\section{Challenges to Drug Test Results}

In situations where there is a challenge to the results of a positive drug test result, the following guidelines must be used. The B sample should be released for analysis to a drug testing laboratory accredited by a recognised external accrediting body and working to these guidelines. This release requires authorisation from both the customer/MRO and the donor.

The release must be supported by chain of custody procedures that can withstand legal scrutiny and include information about the findings of the original test (corresponding A sample) and the cut-offs used for the test. The original laboratory must retain the residue of the original sample and its containers so that it can be compared with the B sample at a later date if required.

All laboratories that undertake $\mathrm{B}$ sample testing must be able to demonstrate that they can accurately determine the concentration of a drug or metabolite at $50 \%$ of the recommended confirmation cut-off concentration listed in Appendix E (or the cut-off used for the original test, whichever is the lower). On receipt in the testing laboratory, the B sample should follow chain of custody procedures as outlined. It is recommended that the laboratory should carry out validity checks outlined prior to carrying out the confirmation analysis. Only those drugs identified for 
confirmation testing should be looked for. The final report on the B sample must say either that there was no drug found, or a named drug was found at a level that is either consistent or inconsistent with the level in the corresponding A sample. Confirmation cut-off levels are not to be used as the determinant. There must be no comment on the final report that states whether the sample is positive or negative.

\section{Appendix A}

\section{Oral Fluid Collection Procedure}

(Note: Only formally competence tested and authorised persons may act as collecting officers. Medical qualification is NOT required for collecting officers.)

\section{Collection Site}

Procedures shall provide for a designated collection site to be secure. During the collection process the collection site must be dedicated solely to sample collection and comply with all local health and safety requirements.

\section{Access to Authorised Personnel Only}

Only authorised personnel shall be permitted in any part of the designated collection site when oral fluid samples are being collected or stored.

\section{Chain of Custody}

During the collection process chain of custody forms will be completed fully by the collecting officer and donor.

\section{Identification of the donor}

When a donor arrives at the collection site, the collecting officer will request that the donor presents photographic identification. If the donor does not have acceptable photographic identification, the collecting officer will obtain a positive identification of the donor by an authorised supervisor or manager within the parent organisation. If the donor's identity cannot be established, the collecting officer will not proceed with the collection.

\section{Informing the donor about the test}

The donor has to be informed about the purpose and the content of the test.

\section{Consent of the donor}

The donor gives his/her consent for oral fluid collection and analysis of drugs by signature. If the donor refuses to give a sample, - a note to this effect should be recorded on the form designated for that purpose. Appendix B gives an example of a Donor’s Statement of Informed Consent.

\section{Privacy}

Procedures for collecting oral fluid specimens shall allow, where possible, for donor privacy during sample collection. Donors have to be treated equally regardless of gender and any physical 
impairment. The process should avoid embarrassment but should also be rugged enough to satisfy challenge of the sample integrity.

If there is a strong suspicion of sample adulteration and/or the previous sample was adulterated the sample can be collected under the supervision of collecting officer.

\section{Integrity of the Specimen}

The collecting officer must adopt procedures to minimise the risk of tampering and adulteration of the specimen during collection. The following minimum precautions shall be taken to ensure that unadulterated specimens are obtained and correctly identified:

a) Throughout the collection process, the collecting officer will note any unusual behaviour of the donor on the chain of custody form.

b) The collecting officer will ask the donor to remove any articles from the mouth e.g. chewing gum.

c) The collecting officer will wait for 10 minutes to observe that the donor has nothing in their mouth.

d) Oral fluid samples will then be collected from the donor and prepared for analysis in strict accordance with the standard operating procedure and the manufacturer's instructions for the collection system being used.

e) Upon receiving the specimen from the donor, the collecting officer will:

- Note collection time

- Check the volume of oral fluid on/in the specimen collection device.

- Inspect the specimen to determine its colour and appearance of any signs of contaminants and adulteration.

f) Any unusual findings will be noted on the chain of custody form.

g) If the volume is less than that required by recommendation/order of the laboratory, the specimen will be discarded and a second specimen will be collected.

h) If the donor is unable to provide a suitable volume of oral fluid for analysis then the collection process is stopped and advice should be sought.

i) Both the donor and the collecting officer will keep the specimen container(s) in view at all times prior to the oral fluid specimen(s) being sealed and labelled.

j) The collecting officer will request the donor to observe the transfer of the specimen to two sample containers and the attachment of the tamper-evident seal to the containers. The same is requested if split samples are obtained by two devices. The tamper-evident seal ensures that any tampering with the specimen will be evident to laboratory personnel during the laboratory receipt.

k) The specimen container(s) will have an identification label that contains at a minimum; the date, the donor's specimen number and the donor's signature/initials. The collecting officer will enter all information on the chain of custody form to identify the origin of the specimen. Both specimen containers and all pages of the chain of custody will be labelled at the time of collection with a unique identifier.

l) The collecting officer will explain the significance relating to the drugs and medicines consumed within a minimum of 7 days prior to the provision of the oral fluid specimen. The donor will be given the opportunity to declare any medication used. 
m) The donor will be asked to read and sign a statement on the chain of custody form certifying that the specimen identified on the form was in fact the specimen provided by the donor and giving informed consent for the work to be undertaken. Appendix B gives an example of a Donor's Statement of Informed Consent.

n) The collecting officer will complete the specimen chain of custody form and package with the oral fluid specimen ready for dispatch together to the analytical laboratory. If the specimen is not dispatched at once, the collecting officer during storage prior to dispatch must give appropriate consideration to the temperature and security of the specimens. It is advised that the specimens should be refrigerated whenever possible (do not freeze).

o) Other pages of the chain of custody form will be given or forwarded to the appropriate persons.

p) The collecting officer and the donor will be present throughout the procedures outlined in the paragraphs of this section.

\section{Exceptional situations}

a. The donor wants to give the sample later

The collecting officer must not allow the donor to leave the collection site and come back later to give a sample. The collecting officer will contact the appropriate authority to obtain guidance on the action to be taken.

b. Admission of illegal drug use

If the donor admits illegal drug use, this should be noted on the chain of custody form.

\section{Transportation to Laboratory}

Collecting officers will arrange to dispatch the collected specimens to the drug-testing laboratory. The specimens will be placed in containers designed to minimise the possibility of damage during shipment and packed properly to comply with local/international mail and courier regulations for biological specimens. Since specimens and the corresponding documents are sealed in packages that would indicate any tampering during transit to the laboratory by couriers, carriers, and postal services, usually there is no requirement for documented chain of custody procedures for the transport of the package.

\section{Appendix B}

\section{Example of a Donor's Statement of Informed Consent}

I confirm that I have received information about the meaning and content of the drug test. I confirm that I have provided a sample of my oral fluid to the specimen collector. I have observed the specimen being placed and sealed in the specimen containers and I confirm that the information on this form and on the specimen labels is correct. I hereby give permission for a minimum of two sealed specimen containers to be sent to the laboratory and I consent that they be tested for evidence of drug use and for tests to be carried out to confirm the validity of the sample. Furthermore, I understand that the results will be communicated confidentially to the employer or a designated representative.

I consent to the above.

Donor's Name (Block Capitals):

Donor's Signature:

Date:

Donor's identifier on the specimen labels

(if different from above): 


\section{Appendix C}

\section{Some Examples of Fatal Flaws in the Chain of Custody}

1. A unique identifier (e.g. barcode) mismatches or absent

2. No documentation received with the sample

3. No written consent to test from the donor

4. Seals broken or tampered with on the sample container/transport container

5. No seals

6. Only 1 sample received

7. Insufficient sample for complete analysis

8. Leaking sample

\section{References}

1. http://www.emcdda.europa.eu/html.cfm/index16901EN.html

2. http://www.un.org/en/universal-declaration-human-rights/

3. Council of Europe: http://www.coe.int/en/web/human-rights-convention/home http://www.echr.coe.int/Documents/Collection_Convention_1950_ENG.pdf

4. http://eur-lex.europa.eu/legal-content/EN/TXT/HTML/?uri=CELEX:31989L0391\&from=EN

5. https://oshwiki.eu/wiki/OSH_system_at_national_level___Italy

Richtlinie der GTFCh zur Qualitätsicherung bei forensisch-toxikologischen

Untersuchungen”Toxichem Krimtech (2009) 76 (3): 142-176

http://www.gtfch.org/cms/images/stories/files/GTFCh_Richtlinie_For-Tox_Version\%201.pdf 